\title{
Role of $\beta$-Arrestins in Endosomal Sorting of G Protein-Coupled Receptors
}

\author{
Rohit Malik ${ }^{1}$ and Adriano Marchese $\mathrm{e}^{1,2, *}$ \\ ${ }^{1}$ Program in Molecular Biology \\ ${ }^{2}$ Department of Molecular Pharmacology and Therapeutics, Loyola University Chicago \\ USA
}

\section{Introduction}

G protein-coupled receptors (GPCRs) are members of a large gene family encoded by approximately 1000 members (Lefkowitz, 2007; Pierce et al., 2002). They are also known as seven transmembrane domain (7TM) receptors as they are characterized by a single polypeptide that has seven membrane spanning helical domains. Members of this family include receptors that bind to biogenic amines, chemokines, opioids, lipids, among many more. They mediate a wide variety of physiological processes ranging from neurotransmission, cardiovascular function, leukocyte chemotaxis and analgesia. GPCRs are important clinically as many have been implicated in many diseases and are targets for approximately $50 \%$ of all marketed medicines (Drews, 2000).

Upon binding to their cognate ligands GPCRs mediate down stream signaling via heterotrimeric GTP-binding proteins (G protein). $G$ proteins are comprised of an $\alpha$-subunit $(G \alpha)$ and a tightly associated dimer of $\beta$ and $\gamma$-subunits $(G \beta \gamma)$. In the inactive state $G \alpha$ is bound to GDP and exists in an inactive conformation. Once the GPCR is activated by its cognate ligand, conformational changes in the receptor induce the exchange of GDP for GTP on G $\alpha$ leading to its activation and dissociation from the $\beta \gamma$ subunits. The activated $\mathrm{G} \alpha(\mathrm{G} \alpha-\mathrm{GTP})$ and dissociated $\beta \gamma$ subunits activate downstream effector molecules contributing to GPCR signaling. One effector molecule is adenylyl cyclase and upon its activation it leads to the production of cyclic AMP (cAMP), which in turn activates the protein kinase A (PKA), a serine/threonine kinase that phosphorylates many different substrates. Another effector molecule that is activated is phospholipase $C$, which catalyzes the hydrolysis of phosphatidyl 4,5 bisphosphate to produce inositol 1,4,5-trisphosphate and diacyclglycerol, which in turn leads to calcium release from intracellular stores and activation of protein kinase $C$, respectively. Recently, it has become apparent that GPCRs may also signal independent of the $G$ protein, and this typically involves proteins known as $\beta$-arrestins (DeWire et al., 2007).

To ensure that signals are of the appropriate magnitude and duration GPCR signaling is tightly regulated. This is critical because perturbations in the regulatory processes that control GPCR signaling may contribute to the cause of human pathologies (Hernandez et al., 2003).

${ }^{*}$ Corresponding Author 
Regulation of signaling is complex and involves multiple steps. It involves inactivation of the $G$ protein and degradation of second messengers (Nelson et al., 2007; Perry et al., 2002). Regulation also involves a series of complex events that occur at the level of the receptor. Regulation may occur by second-messenger-dependent protein kinases PKA and PKC, which may also phosphorylate GPCRs, in addition to other downstream factors, when they become activated. Additional regulation occurs by another family of serine/threonine kinases known as $G$ protein-coupled receptor kinases (GRKs), which phosphorylate activated receptors resulting in the binding of another class of proteins known as arrestins (Fig 1) (Krupnick and Benovic, 1998). Here we will focus on recent advances that have linked non-visual arrestins to a novel function by regulating the endosomal sorting machinery with a role in trafficking of GPCRs into the degradative pathway.

\section{Traditional roles of $\beta$-arrestins}

Mammalian arrestins comprise a family of four members that can be sub-divided into two groups: visual (arrestin-1 and arrestin-4) and non-visual arrestins ( $\beta$-arrestin- 1 and $\beta$ arrestin-2, also known as arrestin-2 and arrestin-3, respectively) (Gurevich and Gurevich, 2006a). Visual arrestins are restricted in distribution to the visual system. Arrestin- 1 is found in high abundance in rod cells whereas arrestin- 4 is found in cone cells. Non-visual arrestins are ubiquitously expressed and likely regulate the signaling of many GPCRs.

The classical known function of arrestins, in conjunction with GRKs, is to mediate GPCR desensitization, a phenomenon in which responsiveness to chronic stimulation is attenuated (Fig. 1). Arrestins were initially identified in the visual system as a $48-\mathrm{kDa}$ protein capable of blocking light induced signaling of rhodopsin (Wilden et al., 1986). Subsequently, $\beta$-arrestins were identified and found to function in non-visual systems in an analogous manner to visual arrestins in that they blocked agonist-induced signaling of the $\beta_{2}$-adrenergic receptor $\left(\beta_{2} \mathrm{AR}\right)$ (Lohse et al., 1990). Arrestins are typically recruited to GPCRs that are activated by their cognate ligands and phosphorylated by GRKs (Krupnick and Benovic, 1998). Arrestin binding uncouples the $G$ protein from the receptor via steric hindrance culminating in attenuated signaling (Lohse et al., 1990). Arrestins may also contribute to signal termination by promoting degradation of second messengers (Perry et al., 2002) (Nelson et al., 2007) and also by promoting GPCR internalization through clathrin-coated pits based on their ability to bind to components of the internalization machinery (Goodman et al., 1996; Laporte et al., 1999).

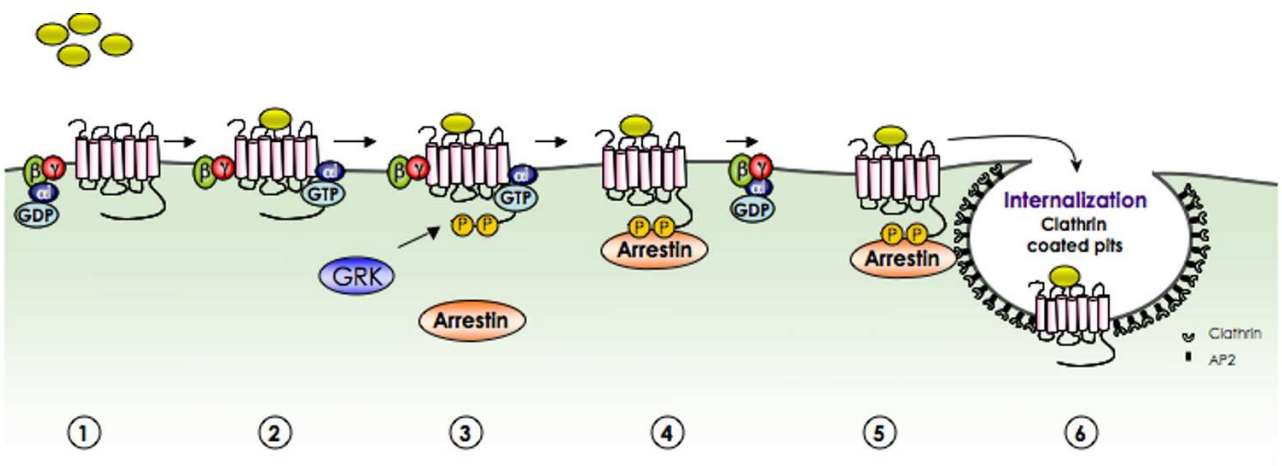

Fig. 1. Schematic depicting $\beta$-arrestin mediated desensitization and internalization of GPCRs. 
1) Under basal conditions, the heterotrimeric GTP binding protein (G protein), which is

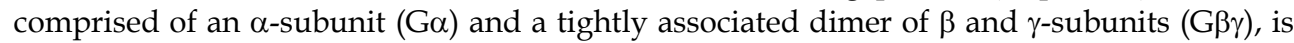
bound to GDP and is in an inactive conformation. 2) Upon binding to its cognate ligand (yellow oval), conformational changes in the GPCR induce the exchange of GDP for GTP on the $\alpha$-subunit $(G \alpha)$ leading to its activation and dissociation from the $\beta \gamma$ subunits. The activated $\alpha$-subunit $(\mathrm{G} \alpha-\mathrm{GTP})$ and $\beta \gamma$ subunits activate downstream effector molecules contributing to GPCR signaling. 3) Signaling is rapidly terminated in part by GPCR kinase (GRK) recruitment to the activated receptor, which phosphorylates the receptor on serine and/or threonine residues that are located within the carboxy-terminal tail and/or on the intracellular loops. 4) Arrestins are rapidly recruited to the phosphorylated receptor, which upon binding uncouple the receptor from the associated $\mathrm{G} \alpha$ subunit via steric hindrance, contributing to signal termination and to the phenomenon known as desensitization. 5) Arrestins subsequently interact with clathrin and AP2, two important components of the internalization machinery. 6) This results in the recruitment of activated and phosphorylated receptors for internalization via clathrin coated pits.

The crystal structure of $\beta$-arrestins revealed that they consist of two distinct elongated domains ( $\mathrm{N}$ and $\mathrm{C}$ domains) and a carboxy-terminal tail (C-tail) (Han et al., 2001; Hirsch et al., 1999; Milano et al., 2002). The $\mathrm{N}$ and $\mathrm{C}$ domains are independent folding units composed of anti-parallel beta sheets connected by a short linker or hinge sequence. Under basal conditions or in an inactive state, the $\mathrm{N}$ and $\mathrm{C}$ domains are compact and the C-tail is anchored to the $\mathrm{N}$ domain. Upon binding to phosphorylated GPCRs arrestins undergo significant conformational changes in which a rearrangement of the $\mathrm{N}$ and $\mathrm{C}$ domains occurs and the C-tail is released (Gurevich and Gurevich, 2006b). The C-tail of $\beta$-arrestins contains the binding sites for clathrin and AP2, two important components of the internalization machinery (Goodman et al., 1996; Laporte et al., 1999). The exposure of the C-tail enables clathrin and AP2 binding and subsequent recruitment of receptors to clathrincoated pits for internalization. The arrestin C-tail also contains sites for post-translational modifications that also regulate the ability of arrestins to promote GPCR internalization. Nitrosylation of $\beta$-arrestin- 2 on the terminal cysteine residue 409 increases its ability to interact with clathrin and facilitate $\beta_{2} \mathrm{AR}$ internalization (Ozawa et al., 2008). SUMOylation of $\beta$-arrestin- 2 on lysine residue 400 has also been shown to have a role in $\beta_{2} \mathrm{AR}$ internalization, likely by facilitating interactions with AP2 (Wyatt et al., 2011). Detailed molecular insight regarding how these events are coordinated to properly execute $\beta$ arrestins roles in GPCR internalization remains to be determined.

\section{Role of $\beta$-arrestins in GPCR recycling}

Once removed from the plasma membrane by internalization receptors are delivered to early endosomes (Hanyaloglu and von Zastrow, 2008; Marchese et al., 2008). Once on endosomes, receptors may enter into the recycling pathway and return to the plasma membrane giving rise to functional resensitization of GPCR responsiveness. Upon agonist activation the $\beta_{2} \mathrm{AR}$ is rapidly phosphorylated and internalized onto endosomes before it is rapidly recycled to the plasma membrane (Krueger et al., 1997; Pippig et al., 1995; von Zastrow and Kobilka, 1992). Internalization onto endosomes may be a prerequisite for receptor resensitization of signaling. The acidic environment of the endosomal compartment may induce a conformational change in the receptor enabling an endosomal associated 
phosphatase to dephosphorylate the receptor (Krueger et al., 1997). Dephosphorylation of the receptor may facilitate its entry into the recycling pathway for delivery to the plasma membrane and functional resensitization.

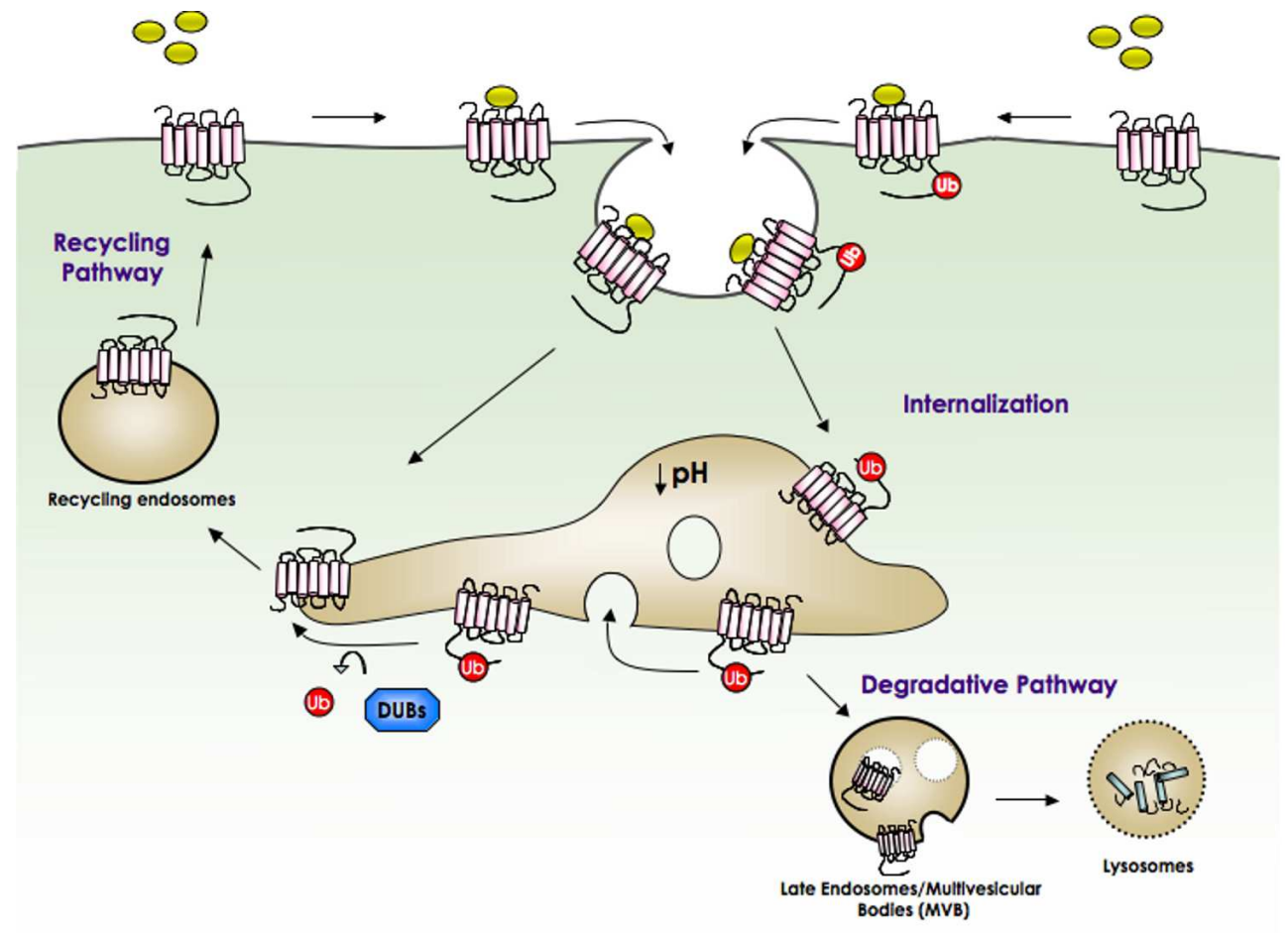

Fig. 2. Trafficking pathways for GPCRs.

Upon activation by its cognate ligand (oval), GPCRs are typically sequestered into specialized microdomains of the plasma membrane that are responsible for endocytosis. These areas of the plasma membrane pinch off, forming vesicles that eventually fuse with early endosomes, where the receptors are delivered. Some receptors may be modified with ubiquitin moieties at the plasma membrane, although the ubiquitin moiety is not required to promote receptor internalization. Once on early endosomes, GPCRs are sorted for either entering the recycling or degradative pathways. GPCRs that enter the degradative pathway are typically modified with ubiquitin, which serves as a signal for sorting into multivesicular bodies (MVBs). MVBs fuse with lysosomes where degradation of the receptor occurs. GPCRs that are not ubiquitinated may enter the recycling pathway and are returned to the plasma membrane via recycling endosomes, giving rise to functional resensitization of signaling. The ubiquitin moieties on some receptors may be removed by the action of deubiquitinating enzymes (DUBs), which may occur on endosomes, and redirect receptors targeted for the degradative pathway into the recycling pathway.

$\beta$-arrestins may also mediate receptor recycling at a post-internalization step by playing a direct role on endosomes. For many GPCRs, the interaction with $\beta$-arrestins is transient. 
Once $\beta$-arrestins bind and desensitization and receptor internalization have initiated, the two proteins disassociate while the receptor internalizes onto endosomes (Oakley et al., 1999; Oakley et al., 2001; Oakley et al., 2000). Once on endosomes the receptor rapidly recycles to the plasma membrane. However, for a subset of GPCRs, not only does $\beta$-arrestin binding promote desensitization and internalization, $\beta$-arrestins may co-internalize and colocalize with the receptor on endosomes. The ability of $\beta$-arrestin to co-internalize with GPCRs is dependent upon their ability to form a stable complex with the GPCR. This slows down the recycling of the receptor, likely by preventing the receptor from efficiently entering into the recycling pathway. Receptors that form a stable interaction with $\beta$-arrestins tend to have multiple serine/threonine residue clusters near the end of their carboxyterminal tail (Oakley et al., 1999; Oakley et al., 2001). Phosphorylation of these clusters promotes high affinity $\beta$-arrestin binding thereby allowing it to remain bound to the receptor while internalization onto endosomes occurs. The stability of the interaction and the trafficking pattern of $\beta$-arrestins also correlates with their ubiquitination status (Shenoy and Lefkowitz, 2003; Shenoy et al., 2009). Upon receptor activation, $\beta$-arrestins may also be subject to ubiquitination (Shenoy et al., 2001). $\beta$-arrestin-2 interacts with the E3 ubiquitin ligase Mdm2 and the deubiquitinating enzyme USP33 (Shenoy et al., 2001; Shenoy et al., 2009). Upon activation of GPCRs for which the interaction with $\beta$-arrestin- 2 is transient, such as $\beta_{2} \mathrm{AR}$, ubiquitination of $\beta$-arrestin- 2 is rapid, but it is rapidly deubiquitinated by USP33. In contrast, upon activation of GPCRs for which the interaction with $\beta$-arrestin-2 is stable, such as the vasopressin V2 receptor, ubiquitination is also rapid, but it is not deubiquitinated by USP33, resulting in sustained ubiquitination. One possible explanation to account for this difference between receptors is that $\beta$-arrestin- 2 may undergo differential conformational changes when bound to distinct receptors which may affect its ability to interact with USP33 (Shenoy et al., 2009). Therefore discrete conformational changes regulated by different GPCRs may control $\beta$-arrestin deubiquitination and thus their ability to co-traffic with receptors onto endosomes.

$\beta$-arrestins may also play a role in GPCR recycling independent of their role in promoting GPCR internalization. Although, $\beta$-arrestins are not required for internalization of the $\mathrm{N}$ formyl peptide receptor (FPR), they are required for promoting FPR recycling. Agonistinduced internalization of FPR occurs in a $\beta$-arrestin-independent fashion, as FPR internalization is not altered in mouse embryonic fibroblasts (MEFs) isolated from $\beta$ arrestin- 1 and $\beta$-arrestin-2 knockout out mice (Vines et al., 2003). However, recycling of the receptor is impaired in the $\beta$-arrestin knock-out MEFs and restored in cells in which $\beta$ arrestin- 1 and $\beta$-arrestin- 2 are re-expressed. Although not required for internalization, agonist activation promotes $\beta$-arrestin binding to FPR and its co-internalization with the receptor onto endosomes. Once on endosomes $\beta$-arrestins promote recycling through a mechanism that remains to be determined.

\section{4. $\beta$-arrestins as adaptors for E3 ubiquitin ligases}

In addition to entering the recycling pathway, GPCRs may be sorted into the degradative pathway (Fig. 2) for delivery to lysosomes where they are degraded (Hanyaloglu and von Zastrow, 2008; Marchese et al., 2008). The functional consequence of this is long-term attenuation of signaling giving rise to a phenomenon known as downregulation. $\beta$ - 
arrestin may serve as an adaptor to recruit E3 ubiquitin ligases directly to activated receptors to mediate their ubiquitination. The ubiquitin moiety serves as a sorting signal on endosomes for targeting the receptor into the degradative pathway. Receptor ubiquitination occurs at the plasma membrane even though the attached ubiquitin serves as a sorting signal on endosomes. Receptor mutants lacking ubiquitination sites internalize normally but are defective in their ability to be sorted or targeted to lysosomes (Marchese and Benovic, 2001; Shenoy et al., 2001). A role for $\beta$-arrestins as an E3 ubiquitin ligase adaptor for receptor ubiquitination was first suggested in studies examining the regulation of $\beta_{2} \mathrm{AR}$ (Shenoy et al., 2001). Agonist-promoted ubiquitination of $\beta_{2} \mathrm{AR}$ is impaired in MEFs isolated from $\beta$-arrestin- 2 knock-out mice, suggesting that $\beta$-arrestin- 2 mediates ubiquitination of $\beta_{2} \mathrm{AR}$. In contrast, $\beta$-arrestin- 1 is not involved in $\beta_{2} \mathrm{AR}$ ubiquitination. $\beta$-arrestin-2 interacts with the HECT-domain E3 ubiquitin ligase Nedd4, which mediates ubiquitination of $\beta_{2} \mathrm{AR}$ (Shenoy et al., 2008). Although $\beta$-arrestin-2 interacts with Mdm2, it does not mediate ubiquitination of $\beta_{2} \mathrm{AR}$ (Shenoy et al., 2001). Depletion of Nedd 4 by siRNA attenuates $\beta_{2}$ AR ubiquitination and lysosomal targeting and the interaction between $\beta_{2} \mathrm{AR}$ and Nedd4 is dependent upon the presence of $\beta$ arrestin-2 (Shenoy et al., 2008). This is consistent for a role of $\beta$-arrestin-2 serving as an adaptor to recruit Nedd4 to $\beta_{2} \mathrm{AR}$.

Alternatively, Nedd 4 may be recruited to $\beta_{2} \mathrm{AR}$ independent of $\beta$-arrestin- 2 involving the arrestin domain-containing protein ARRDC3 (Nabhan et al., 2010). ARRDs are a family of 6 mammalian proteins related to yeast proteins called arrestin-related trafficking (ART) adaptor proteins that were first characterized in Saccharomyces cerevisiae (Lin et al., 2008). Collectively these proteins have been referred to as alpha-arrestins to distinguish them from $\beta$-arrestins (Alvarez, 2008). Although sharing very little amino acid sequence identity with mammalian $\beta$-arrestins, bioinformatics modeling revealed that alphaarrestins have an arrestin-fold consisting of $\mathrm{N}$ and $\mathrm{C}$ domains. This overall architecture may represent a conserved structural design found in seemingly distantly related proteins. The retromer component Vps26, although it does not share primary sequence identity with mammalian arrestins, has structurally similar folded $\mathrm{N}$ and $\mathrm{C}$ domains (Shi et al., 2006). One key distinguishing feature of ARRDs compared to $\beta$-arrestins or visual arrestins is that ARRDs have a long carboxy-terminal region harboring PY motifs (Lin et al., 2008). PY motifs are short stretches of amino acids typically found in the context of PP $x Y$ and PPPY, where $\mathrm{P}$ is a proline residue, $x$ is any amino acid and $\mathrm{Y}$ is a tyrosine residue (Einbond and Sudol, 1996). PY motifs are typically recognized by WW domains, which are domains of approximately 30 amino acids containing two highly conserved tryptophan residues (Macias et al., 2002). The Nedd4-like family of HECT-domain E3 ubiquitin ligases, comprised of 9 members in the human genome, is characterized by the presence of 4 tandemly linked WW domains (Ingham et al., 2004). Substrates that have PY motifs interact directly with their cognate E3s via the WW domains, however, many substrates do not have PY motifs and are believed to interact indirectly with these E3s through an adaptor protein that harbors a PY motif (Shearwin-Whyatt et al., 2006). Recently, it was reported that ARRDC3 interacts with $\beta_{2} \mathrm{AR}$ and serves as an adaptor for Nedd4-dependent ubiquitination of the receptor (Nabhan et al., 2010). Depletion of ARRDC3 attenuates agonist-induced ubiquitination and lysosomal targeting of $\beta_{2} \mathrm{AR}$ (Nabhan et al., 2010). ARRDC3 is predicted to bind directly to Nedd4 via its PY motif and 
to $\beta_{2} \mathrm{AR}$. Mammalian arrestins do not contain PY motifs and it is unclear how Nedd4 interacts with $\beta$-arrestin-2. It remains to be determined how the actions of $\beta$-arrestin- 2 and ARRDC3 are coordinated to mediate Nedd4-dependent $\beta_{2}$ AR ubiquitination.

Interestingly, $\beta$-arrestin-1 interacts with another member of the Nedd4-like family of E3 ubiquitin ligases known as AIP4 (Bhandari et al., 2007). AIP4 mediates ubiquitination and endosomal sorting of the GPCR chemokine receptor C-X-C receptor 4 (CXCR4) (Marchese et al., 2003). As with all members of this family, AIP4 has a C2 domain, 4 tandemly-linked WW domains and the catalytic HECT domain. Unlike Nedd4, however, AIP4 has a proline-rich region that may bind to a subset of SH3 domains (Angers et al., 2004). The interaction between $\beta$-arrestin- 1 and AIP4 is direct and it is mediated by AIP4 WW domains I and II, not III and IV (Bhandari et al., 2007). In addition to interacting with PY motifs, WW domains are also known to interact with phosphorylated serine or threonine residues in the presence of an adjacent proline residue (p(S/T)P) (Lu et al., 1999; Verdecia et al., 2000). Presently, it remains to be determined how the AIP4 WW-domains interact with $\beta$-arrestin-1. Interestingly, a non-canonical WW-mediated interaction involving AIP4 has been recently reported (Bhandari et al., 2009). The WW domains of AIP4 have been shown to interact with phosphorylated serine residues in the absence of nearby proline residues. Phosphorylation of serine residues within the C-tail of CXCR4 mediates a direct interaction with AIP4 via WW domains I and II (Bhandari et al., 2009). A receptor mutant in which C-tail serine residues 324 and 325 were changed to alanine residues (S324/5A) attenuated binding to AIP4 (Bhandari et al., 2009; Marchese and Benovic, 2001). Accordingly, the S324/5A CXCR4 receptor mutant shows defective ubiquitination and degradation (Bhandari et al., 2009). Agonist-promoted phosphorylation of these residues occurs at the plasma membrane leading to AIP4 recruitment to the plasma membrane where the receptor is ubiquitinated. Therefore for CXCR4 ubiquitination, an adaptor is not required because the Nedd4-like E3 AIP4 can interact directly with the receptor. Similar to the mechanism of the interaction between AIP4 and CXCR4, the WW domains of Nedd4 may also interact with its substrates via phosphorylated serine residues in the absence of nearby proline residues (Edwin et al., 2010). Therefore, phosphorylated serine residues in the absence of proline residues may represent a general recognition motif for WW domains. To our knowledge, Nedd4 and AIP4 are the only Nedd4-like E3 ubiquitin ligases assigned to the ubiquitination of mammalian GPCRs, but it is tempting to speculate that CXCR4 recognition by AIP4 may represent a general mechanism by which Nedd4-like E3 family members interact with and ubiquitinate GPCRs.

\section{5. $\beta$-arrestins as adaptors for endosomal sorting}

Although $\beta$-arrestin- 1 interacts with AIP4 it is not involved in ubiquitination of CXCR4. Depletion of $\beta$-arrestin-1 by siRNA does not block CXCR4 ubiquitination and nor does it block CXCR4 internalization (Bhandari et al., 2007). However, $\beta$-arrestin- 1 depletion blocks CXCR4 degradation by preventing its trafficking from early to late endosomes, suggesting a role for $\beta$-arrestin- 1 at a sorting step on endosomes (Bhandari et al., 2007). AIP4 also mediates sorting of CXCR4 on endosomes, in addition to its role at the plasma membrane in mediating CXCR4 ubiquitination (Marchese et al., 2003). Both $\beta$-arrestin- 1 and AIP4 colocalize with CXCR4 on early endosomes where they likely function to sort CXCR4 into the degradative pathway. Therefore $\beta$-arrestin- 1 acts on early endosomes as an endosomal 
adaptor molecule to regulate the trafficking of ubiquitinated CXCR4 from early endosomes to lysosomes (Bhandari et al., 2007; Malik and Marchese, 2010).

One function of endosomal $\beta$-arrestin- 1 is likely to recruit ubiquitinated CXCR4 to the endosomal sorting required for transport (ESCRT) pathway, the degradative pathway that targets ubiquitinated receptors for lysosomal degradation [Fig. 3 and (Malik and Marchese, 2010)]. The ESCRT machinery consists of four discrete multi-protein complexes named ESCRT 0-III (Henne et al., 2011). The ESCRT complexes act in a sequential and a coordinated manner to target ubiquitinated cargo into intraluminal vesicles (ILVs) of multivesicular bodies, which then fuse with lysosomes where degradation occurs. Delivery into the ESCRT pathway is thought to occur when the ubiquitin moiety on ubiquitinated receptors is initially recognized by the ubiquitin binding domains (UBD) found in ESCRT 0 (Raiborg and Stenmark, 2009; Shields et al., 2009). ESCRT-0 subsequently recruits ESCRT-I to the endosomal membrane, followed by recruitment of ESCRT II and III, culminating in sorting of the receptor into ILVs [reviewed in (Raiborg and Stenmark, 2009)]. ESCRT-0 is comprised of two proteins: HRS (hepatocyte growth factor-regulated tyrosine kinase substrate) and STAM (signal transduction adaptor molecule). $\beta$-arrestin-1 interacts with ESCRT-0 through a direct interaction with STAM-1 (Malik and Marchese, 2010). Therefore it is possible that $\beta$-arrestin- 1 facilitates ubiquitinated CXCR4 recruitment to ESCRT-0 for entry into the ESCRT pathway.

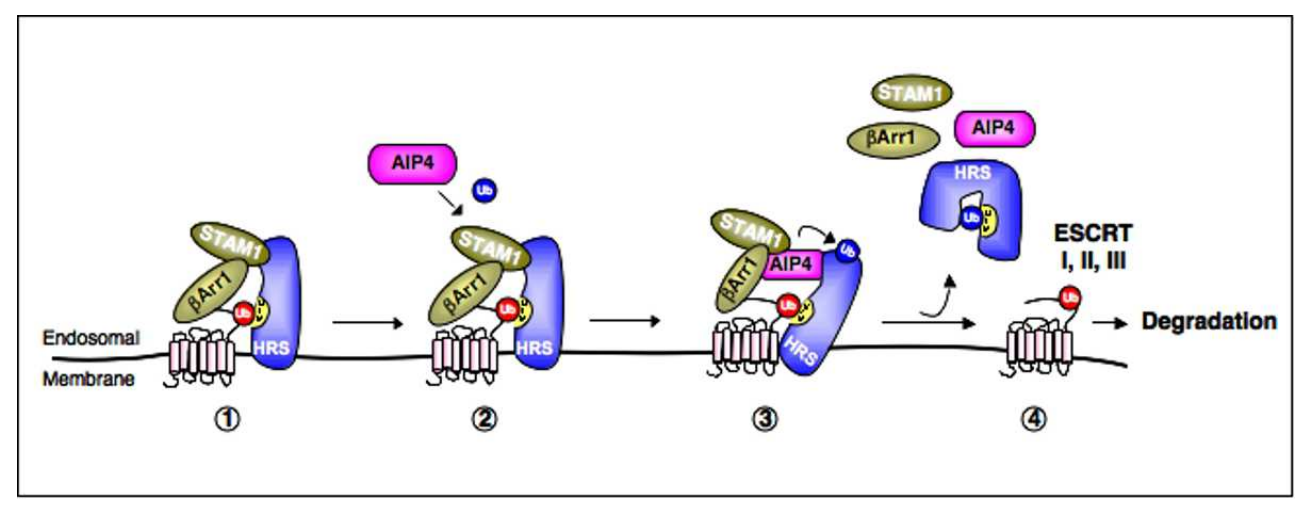

Fig. 3. Proposed model for the roles of $\beta$-arrestins in endosomal sorting of CXCR4.

1) $\beta$-arrestin-1 ( $\beta$ Arr1) interacts with ESCRT-0 through a direct interaction with STAM-1. STAM-1 together with HRS form the core components of ESCRT-0. Although the ubiquitin moiety on CXCR4 may be recognized by the ubiquitin interacting motif (UIM) present in HRS, one role of $\beta$-arrestin-1 may be to facilitate the interaction of ubiquitinated CXCR4 with ESCRT-0. 2) A second role for $\beta$-arrestin-1 in conjunction with STAM-1 may serve to recruit the E3 ubiquitin ligase AIP4 for ubiquitination of HRS. 3) The ubiquitin moiety on HRS may induce an auto-inhibitory conformation through an interaction with its UIM domain to relieve HRS of its sorting function. 4) The precise molecular details remain unknown, but this somehow leads to the delivery of 
ubiquitinated CXCR4 to downstream elements of the ESCRT machinery for subsequent entry into ILVs and delivery to lysosomes for degradation.

Surprisingly, depletion of STAM-1 by siRNA accelerates CXCR4 degradation, indicating that STAM-1 negatively regulates CXCR4 entry into the degradative pathway (Malik and Marchese, 2010). This function of STAM- 1 is dependent on the interaction with $\beta$-arrestin1 as disruption of this interaction by expressing fragments encoding the minimal binding regions of each respective protein also accelerates CXCR4 degradation. Although detailed mechanistic insight is lacking, the function of the STAM- $1 / \beta$-arrestin- 1 interaction may be to regulate the ubiquitination status of HRS. Disruption of the STAM- $1 / \beta$-arrestin- 1 interaction decreases CXCR4-induced ubiquitination HRS, indicating that STAM- $1 / \beta-$ arrestin- 1 function to mediate HRS ubiquitination. As $\beta$-arrestin- 1 interacts with AIP4, it may be that together with STAM-1 they function as adaptors for recruiting AIP4 to endosomes to mediate ubiquitination of HRS. This suggests that ubiquitination of HRS serves to delay endosomal sorting of CXCR4. One possible explanation is that the ubiquitin moiety on HRS may interact with its own UIM, a type of ubiquitin binding domain, resulting in an auto-inhibitory conformation that inhibits the ability of HRS to perform its sorting function (Hoeller et al., 2006). Alternatively, the ubiquitin moiety on HRS may be required to interact with other ubiquitin binding domains present in other proteins of the ESCRT machinery and possibly other factors. Regardless, this may represent a way in which receptors can regulate their own sorting efficiency (Malik and Marchese, 2010).

Remarkably, linking GPCRs to the ESCRT machinery may be an evolutionary conserved function of $\beta$-arrestins (Herrador et al., 2010; Herranz et al., 2005). Rim8, an arrestin-like molecule in Saccharomyces cerevisiae related to PalF, an arrestin-like molecule in the fungus Aspergillus nidulans, interacts with components of the ESCRT machinery (Herrador et al., 2010). Sequence homology predicts that Rim8 and PalF share a limited amount of sequence identity with mammalian arrestins. In fungi, PalF may interact with a putative seven transmembrane domain (7TM) receptor in an analogous manner to which $\beta$ arrestins interact with ligand activated GPCRs (Herranz et al., 2005). Rim8 and PalF are involved in a signaling cascade that senses the $\mathrm{pH}$ of the environment. In fungi, $\mathrm{pH}$ is recognized in part by the putative 7TM receptor called PalH. Akaline $\mathrm{pH}$ is thought to activate $\mathrm{PalH}$ and to promote its binding to PalF. The predicted cytoplasmic tail of $\mathrm{PalH}$ interacts with PalF and this interaction may be necessary to activate the intracellular signaling pathway necessary for $\mathrm{pH}$ sensing. Intriguingly, genetic screens have revealed that components of the ESCRT machinery, including ESCRT-I, ESCRT-II and ESCRT-III subunits Snf7 and Vps20, but not ESCRT-0 and ESCRT-III subunits Vps2 and Vps24, are also necessary for $\mathrm{pH}$ sensing ( $\mathrm{Xu}$ et al., 2004). Rim8, the orthologue of PalF binds to Rim21, a 7TM receptor with $\mathrm{pH}$ sensing capabilities (Herrador et al., 2010). Not only does the arrestin-like molecule Rim8 interact with the receptor Rim21 it also interacts with the ESCRT machinery. Rim8 interacts with ESCRT-I subunits Vps23 and Vps28 and as genetic evidence suggests that ESCRT-0 is not involved in $\mathrm{pH}$ sensing signaling ( $\mathrm{Xu}$ et al., 2004), therefore the arrestin-like protein Rim8 may link the 7TM receptor Rim21 directly to ESCRT-I. This raises the intriguing possibility that arrestin recruitment of GPCRs to the ESCRT machinery may represent a conserved function. 


\section{Conclusion}

In addition to their roles in GPCR desensitization, internalization and recycling, $\beta$-arrestins may also function in GPCR endosomal sorting. In this capacity they may serve to link ubiquitinated GPCRs to the ESCRT machinery for subsequent targeting to lysosomes. This may represent an evolutionary conserved function of $\beta$-arrestins, suggesting they may play a broad role in GPCR endosomal sorting. Further studies are required to gain greater mechanistic insight into the process by which $\beta$-arrestins integrate with the ESCRT machinery to target GPCRs for lysosomal degradation.

\section{Acknowledgments}

Grants from the National Institutes of Health (GM075159 and DA026040 to A.M.) supported this work. R.M. was supported in part by a predoctoral fellowship from the American Heart Association (0910098G).

\section{References}

Angers, A., Ramjaun, A.R., and McPherson, P.S. (2004). The HECT domain ligase itch ubiquitinates endophilin and localizes to the trans-Golgi network and endosomal system. J Biol Chem. 279 (12), pp.11471-11479.

Bhandari, D., Robia, S.L., and Marchese, A. (2009). The E3 ubiquitin ligase atrophin interacting protein 4 binds directly to the chemokine receptor CXCR4 via a novel WW domain-mediated interaction. Mol Biol Cell. 20 (5), pp.1324-1339.

Bhandari, D., Trejo, J., Benovic, J.L., and Marchese, A. (2007). Arrestin-2 interacts with the ubiquitin-protein isopeptide ligase atrophin-interacting protein 4 and mediates endosomal sorting of the chemokine receptor CXCR4. J Biol Chem. 282 (51), pp.36971-36979.

DeWire, S.M., Ahn, S., Lefkowitz, R.J., and Shenoy, S.K. (2007). Beta-arrestins and cell signaling. Annu Rev Physiol. 69 pp.483-510.

Drews, J. (2000). Drug discovery: a historical perspective. Science. 287 (5460), pp.1960-1964.

Edwin, F., Anderson, K., and Patel, T.B. (2010). HECT domain-containing E3 ubiquitin ligase Nedd4 interacts with and ubiquitinates Sprouty2. J Biol Chem. 285 (1), pp.255-264.

Einbond, A., and Sudol, M. (1996). Towards prediction of cognate complexes between the WW domain and proline-rich ligands. FEBS Lett. 384 (1), pp.1-8.

Goodman, O.B., Jr., Krupnick, J.G., Santini, F., Gurevich, V.V., Penn, R.B., Gagnon, A.W., Keen, J.H., and Benovic, J.L. (1996). Beta-arrestin acts as a clathrin adaptor in endocytosis of the beta2-adrenergic receptor. Nature. 383 (6599), pp.447-450.

Gurevich, E.V., and Gurevich, V.V. (2006a). Arrestins: ubiquitous regulators of cellular signaling pathways. Genome Biol. 7 (9), pp.236.

Gurevich, V.V., and Gurevich, E.V. (2006b). The structural basis of arrestin-mediated regulation of G-protein-coupled receptors. Pharmacol Ther. 110 (3), pp.465-502. 
Han, M., Gurevich, V.V., Vishnivetskiy, S.A., Sigler, P.B., and Schubert, C. (2001). Crystal structure of beta-arrestin at 1.9 A: possible mechanism of receptor binding and membrane Translocation. Structure. 9 (9), pp.869-880.

Hanyaloglu, A.C., and von Zastrow, M. (2008). Regulation of GPCRs by endocytic membrane trafficking and its potential implications. Annu Rev Pharmacol Toxicol. 48 pp.537-568.

Henne, W.M., Buchkovich, N.J., and Emr, S.D. (2011). The ESCRT pathway. Dev Cell. 21 (1), pp.77-91.

Hernandez, P.A., Gorlin, R.J., Lukens, J.N., Taniuchi, S., Bohinjec, J., Francois, F., Klotman, M.E., and Diaz, G.A. (2003). Mutations in the chemokine receptor gene CXCR4 are associated with WHIM syndrome, a combined immunodeficiency disease. Nat Genet. 34 (1), pp.70-74.

Herrador, A., Herranz, S., Lara, D., and Vincent, O. (2010). Recruitment of the ESCRT machinery to a putative seven-transmembrane-domain receptor is mediated by an arrestin-related protein. Mol Cell Biol. 30 (4), pp.897-907.

Herranz, S., Rodriguez, J.M., Bussink, H.J., Sanchez-Ferrero, J.C., Arst, H.N., Jr., Penalva, M.A., and Vincent, O. (2005). Arrestin-related proteins mediate $\mathrm{pH}$ signaling in fungi. Proc Natl Acad Sci U S A. 102 (34), pp.12141-12146.

Hirsch, J.A., Schubert, C., Gurevich, V.V., and Sigler, P.B. (1999). The 2.8 A crystal structure of visual arrestin: a model for arrestin's regulation. Cell. 97 (2), pp.257269.

Hoeller, D., Crosetto, N., Blagoev, B., Raiborg, C., Tikkanen, R., Wagner, S., Kowanetz, K., Breitling, R., Mann, M., Stenmark, H., and Dikic, I. (2006). Regulation of ubiquitin-binding proteins by monoubiquitination. Nat Cell Biol. 8 (2), pp.163-169.

Ingham, R.J., Gish, G., and Pawson, T. (2004). The Nedd4 family of E3 ubiquitin ligases: functional diversity within a common modular architecture. Oncogene. 23 (11), pp.1972-1984.

Krueger, K.M., Daaka, Y., Pitcher, J.A., and Lefkowitz, R.J. (1997). The role of sequestration in $\mathrm{G}$ protein-coupled receptor resensitization. Regulation of beta2adrenergic receptor dephosphorylation by vesicular acidification. J Biol Chem. 272 (1), pp.5-8.

Krupnick, J.G., and Benovic, J.L. (1998). The role of receptor kinases and arrestins in G protein-coupled receptor regulation. Annu Rev Pharmacol Toxicol. 38 pp.289-319.

Laporte, S.A., Oakley, R.H., Zhang, J., Holt, J.A., Ferguson, S.S., Caron, M.G., and Barak, L.S. (1999). The beta2-adrenergic receptor/betaarrestin complex recruits the clathrin adaptor AP-2 during endocytosis. Proc Natl Acad Sci U S A. 96 (7), pp.3712-3717.

Lefkowitz, R.J. (2007). Seven transmembrane receptors: something old, something new. Acta Physiol (Oxf). 190 (1), pp.9-19.

Lin, C.H., MacGurn, J.A., Chu, T., Stefan, C.J., and Emr, S.D. (2008). Arrestin-related ubiquitin-ligase adaptors regulate endocytosis and protein turnover at the cell surface. Cell. 135 (4), pp.714-725. 
Lohse, M.J., Benovic, J.L., Codina, J., Caron, M.G., and Lefkowitz, R.J. (1990). betaArrestin: a protein that regulates beta-adrenergic receptor function. Science. 248 (4962), pp.1547-1550.

Lu, P.J., Zhou, X.Z., Shen, M., and Lu, K.P. (1999). Function of WW domains as phosphoserine- or phosphothreonine-binding modules. Science. 283 (5406), pp.1325-1328.

Macias, M.J., Wiesner, S., and Sudol, M. (2002). WW and SH3 domains, two different scaffolds to recognize proline-rich ligands. FEBS Lett. 513 (1), pp.30-37.

Malik, R., and Marchese, A. (2010). Arrestin-2 interacts with the endosomal sorting complex required for transport machinery to modulate endosomal sorting of CXCR4. Mol Biol Cell. 21 (14), pp.2529-2541.

Marchese, A., and Benovic, J.L. (2001). Agonist-promoted ubiquitination of the G proteincoupled receptor CXCR4 mediates lysosomal sorting. J Biol Chem. 276 (49), pp.45509-45512.

Marchese, A., Paing, M.M., Temple, B.R., and Trejo, J. (2008). G protein-coupled receptor sorting to endosomes and lysosomes. Annu Rev Pharmacol Toxicol. 48 pp.601-629.

Marchese, A., Raiborg, C., Santini, F., Keen, J.H., Stenmark, H., and Benovic, J.L. (2003). The E3 ubiquitin ligase AIP4 mediates ubiquitination and sorting of the G protein-coupled receptor CXCR4. Dev Cell. 5 (5), pp.709-722.

Milano, S.K., Pace, H.C., Kim, Y.M., Brenner, C., and Benovic, J.L. (2002). Scaffolding functions of arrestin-2 revealed by crystal structure and mutagenesis. Biochemistry. 41 (10), pp.3321-3328.

Nabhan, J.F., Pan, H., and Lu, Q. (2010). Arrestin domain-containing protein 3 recruits the NEDD4 E3 ligase to mediate ubiquitination of the beta2-adrenergic receptor. EMBO Rep. 11 (8), pp.605-611.

Nelson, C.D., Perry, S.J., Regier, D.S., Prescott, S.M., Topham, M.K., and Lefkowitz, R.J. (2007). Targeting of diacylglycerol degradation to M1 muscarinic receptors by beta-arrestins. Science. 315 (5812), pp.663-666.

Oakley, R.H., Laporte, S.A., Holt, J.A., Barak, L.S., and Caron, M.G. (1999). Association of beta-arrestin with $G$ protein-coupled receptors during clathrin-mediated endocytosis dictates the profile of receptor resensitization. J Biol Chem. 274 (45), pp.32248-32257.

Oakley, R.H., Laporte, S.A., Holt, J.A., Barak, L.S., and Caron, M.G. (2001). Molecular determinants underlying the formation of stable intracellular $G$ protein-coupled receptor-beta-arrestin complexes after receptor endocytosis*. J Biol Chem. 276 (22), pp.19452-19460.

Oakley, R.H., Laporte, S.A., Holt, J.A., Caron, M.G., and Barak, L.S. (2000). Differential affinities of visual arrestin, beta arrestin1, and beta arrestin2 for G proteincoupled receptors delineate two major classes of receptors. J Biol Chem. 275 (22), pp.17201-17210.

Ozawa, K., Whalen, E.J., Nelson, C.D., Mu, Y., Hess, D.T., Lefkowitz, R.J., and Stamler, J.S. (2008). S-nitrosylation of beta-arrestin regulates beta-adrenergic receptor trafficking. Mol Cell. 31 (3), pp.395-405. 
Perry, S.J., Baillie, G.S., Kohout, T.A., McPhee, I., Magiera, M.M., Ang, K.L., Miller, W.E., McLean, A.J., Conti, M., Houslay, M.D., and Lefkowitz, R.J. (2002). Targeting of cyclic AMP degradation to beta 2-adrenergic receptors by beta-arrestins. Science. 298 (5594), pp.834-836.

Pierce, K.L., Premont, R.T., and Lefkowitz, R.J. (2002). Seven-transmembrane receptors. Nat Rev Mol Cell Biol. 3 (9), pp.639-650.

Pippig, S., Andexinger, S., and Lohse, M.J. (1995). Sequestration and recycling of beta 2adrenergic receptors permit receptor resensitization. Mol Pharmacol. 47 (4), pp.666-676.

Raiborg, C., and Stenmark, H. (2009). The ESCRT machinery in endosomal sorting of ubiquitylated membrane proteins. Nature. 458 (7237), pp.445-452.

Shearwin-Whyatt, L., Dalton, H.E., Foot, N., and Kumar, S. (2006). Regulation of functional diversity within the Nedd4 family by accessory and adaptor proteins. Bioessays. 28 (6), pp.617-628.

Shenoy, S.K., and Lefkowitz, R.J. (2003). Trafficking patterns of beta-arrestin and G protein-coupled receptors determined by the kinetics of beta-arrestin deubiquitination. J Biol Chem. 278 (16), pp.14498-14506.

Shenoy, S.K., McDonald, P.H., Kohout, T.A., and Lefkowitz, R.J. (2001). Regulation of receptor fate by ubiquitination of activated beta 2-adrenergic receptor and betaarrestin. Science. 294 (5545), pp.1307-1313.

Shenoy, S.K., Modi, A.S., Shukla, A.K., Xiao, K., Berthouze, M., Ahn, S., Wilkinson, K.D., Miller, W.E., and Lefkowitz, R.J. (2009). Beta-arrestin-dependent signaling and trafficking of 7-transmembrane receptors is reciprocally regulated by the deubiquitinase USP33 and the E3 ligase Mdm2. Proc Natl Acad Sci U S A. 106 (16), pp.6650-6655.

Shenoy, S.K., Xiao, K., Venkataramanan, V., Snyder, P.M., Freedman, N.J., and Weissman, A.M. (2008). Nedd4 mediates agonist-dependent ubiquitination, lysosomal targeting, and degradation of the beta2-adrenergic receptor. J Biol Chem. 283 (32), pp.22166-22176.

Shi, H., Rojas, R., Bonifacino, J.S., and Hurley, J.H. (2006). The retromer subunit Vps26 has an arrestin fold and binds Vps35 through its C-terminal domain. Nat Struct Mol Biol. 13 (6), pp.540-548.

Shields, S.B., Oestreich, A.J., Winistorfer, S., Nguyen, D., Payne, J.A., Katzmann, D.J., and Piper, R. (2009). ESCRT ubiquitin-binding domains function cooperatively during MVB cargo sorting. J Cell Biol. 185 (2), pp.213-224.

Verdecia, M.A., Bowman, M.E., Lu, K.P., Hunter, T., and Noel, J.P. (2000). Structural basis for phosphoserine-proline recognition by group IV WW domains. Nat Struct Biol. 7 (8), pp.639-643.

Vines, C.M., Revankar, C.M., Maestas, D.C., LaRusch, L.L., Cimino, D.F., Kohout, T.A., Lefkowitz, R.J., and Prossnitz, E.R. (2003). N-formyl peptide receptors internalize but do not recycle in the absence of arrestins. J Biol Chem. 278 (43), pp.4158141584. 
von Zastrow, M., and Kobilka, B.K. (1992). Ligand-regulated internalization and recycling of human beta 2-adrenergic receptors between the plasma membrane and endosomes containing transferrin receptors. J Biol Chem. 267 (5), pp.3530-3538.

Wilden, U., Hall, S.W., and Kuhn, H. (1986). Phosphodiesterase activation by photoexcited rhodopsin is quenched when rhodopsin is phosphorylated and binds the intrinsic 48-kDa protein of rod outer segments. Proc Natl Acad Sci U S A. 83 (5), pp.11741178.

Wyatt, D., Malik, R., Vesecky, A.C., and Marchese, A. (2011). Small ubiquitin-like modifier modification of arrestin-3 regulates receptor trafficking. J Biol Chem. 286 (5), pp.3884-3893.

Xu, W., Smith, F.J., Jr., Subaran, R., and Mitchell, A.P. (2004). Multivesicular body-ESCRT components function in $\mathrm{pH}$ response regulation in Saccharomyces cerevisiae and Candida albicans. Mol Biol Cell. 15 (12), pp.5528-5537. 


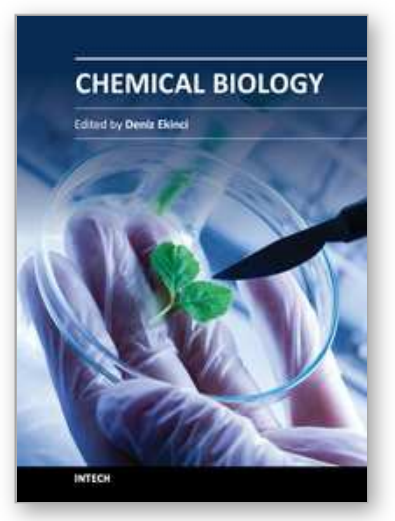

\author{
Chemical Biology \\ Edited by Prof. Deniz Ekinci
}

ISBN 978-953-51-0049-2

Hard cover, 444 pages

Publisher InTech

Published online 17, February, 2012

Published in print edition February, 2012

Chemical biology utilizes chemical principles to modulate systems to either investigate the underlying biology or create new function. Over recent years, chemical biology has received particular attention of many scientists in the life sciences from botany to medicine. This book contains an overview focusing on the research area of protein purification, enzymology, vitamins, antioxidants, biotransformation, gene delivery, signaling, regulation and organization. Particular emphasis is devoted to both theoretical and experimental aspects. The textbook is written by international scientists with expertise in synthetic chemistry, protein biochemistry, enzymology, molecular biology, drug discovery and genetics many of which are active chemical, biochemical and biomedical research. The textbook is expected to enhance the knowledge of scientists in the complexities of chemical and biological approaches and stimulate both professionals and students to dedicate part of their future research in understanding relevant mechanisms and applications of chemical biology.

\title{
How to reference
}

In order to correctly reference this scholarly work, feel free to copy and paste the following:

Rohit Malik and Adriano Marchese (2012). Role of $\beta$-Arrestins in Endosomal Sorting of G Protein-Coupled Receptors, Chemical Biology, Prof. Deniz Ekinci (Ed.), ISBN: 978-953-51-0049-2, InTech, Available from: http://www.intechopen.com/books/chemical-biology/role-of-arrestins-in-endosomal-sorting-of-g-proteincoupled-receptors

\section{INTECH}

open science | open minds

\author{
InTech Europe \\ University Campus STeP Ri \\ Slavka Krautzeka 83/A \\ 51000 Rijeka, Croatia \\ Phone: +385 (51) 770447 \\ Fax: +385 (51) 686166 \\ www.intechopen.com
}

\author{
InTech China \\ Unit 405, Office Block, Hotel Equatorial Shanghai \\ No.65, Yan An Road (West), Shanghai, 200040, China \\ 中国上海市延安西路65号上海国际贵都大饭店办公楼 405 单元 \\ Phone: +86-21-62489820 \\ Fax: $+86-21-62489821$
}


(C) 2012 The Author(s). Licensee IntechOpen. This is an open access article distributed under the terms of the Creative Commons Attribution 3.0 License, which permits unrestricted use, distribution, and reproduction in any medium, provided the original work is properly cited. 